\title{
A pulsed-laser calibration system for the laser backscatter diagnostics at the Omega laser
}

P. Neumayer, C. Sorce, D. H. Froula, V. Rekow, K. Loughman, R. Knight, S. H. Glenzer, R. Bahr, W. Seka

May 7, 2008

High Temperature Plasma Diagnostics Albuquerque, NM, United States May 11, 2008 through May 15, 2008 
This document was prepared as an account of work sponsored by an agency of the United States government. Neither the United States government nor Lawrence Livermore National Security, LLC, nor any of their employees makes any warranty, expressed or implied, or assumes any legal liability or responsibility for the accuracy, completeness, or usefulness of any information, apparatus, product, or process disclosed, or represents that its use would not infringe privately owned rights. Reference herein to any specific commercial product, process, or service by trade name, trademark, manufacturer, or otherwise does not necessarily constitute or imply its endorsement, recommendation, or favoring by the United States government or Lawrence Livermore National Security, LLC. The views and opinions of authors expressed herein do not necessarily state or reflect those of the United States government or Lawrence Livermore National Security, LLC, and shall not be used for advertising or product endorsement purposes. 


\title{
A pulsed-laser calibration system for the laser backscatter diagnostics at the Omega laser
}

Paul Neumayer, Charles Sorce, Dustin H Froula, Vern Rekow, Kevin Loughman, Russel

\author{
Knight, and Siegfried H Glenzer \\ Lawrence Livermore National Laboratory \\ Raymond Bahr and Wolf Seka \\ Laboratory for Laser Energetics
}

A calibration system has been developed that allows a direct determination of the sensitivity of the laser backscatter diagnostics at the Omega laser ${ }^{1}$. A motorized mirror at the target location redirects individual pulses of a mJ-class laser onto the diagnostic to allow the in-situ measurement of the local point response of the backscatter diagnostics. Featuring dual wavelength capability at the $2^{\text {nd }}$ and $3^{\text {rd }}$ harmonic of the Nd:YAG laser, both spectral channels of the backscatter diagnostics can be directly calibrated. In addition, channel cross-talk and polarization sensitivity can be determined. The calibration system has been employed repeatedly over the last two years and has enabled precise backscatter measurements of both stimulated Brillouin scattering and stimulated Raman scattering in gas-filled hohlraum targets that emulate conditions relevant to those in inertial confinement fusion targets.

52.40.Nk, 52.58.Ns 


\section{Introduction}

In the indirect-drive approach to inertial confinement fusion the deuterium-tritium fuel is compressed by the ablation pressure of soft $\mathrm{x}$-rays inside a laser-heated high-Z cavity (hohlraum $)^{2}$. This requires intense laser pulses at wavelengths of the $2^{\text {nd }}$ or $3^{\text {rd }}$ harmonic of $\mathrm{Nd}$ :glass to propagate over distances of several $\mathrm{mm}$ before depositing their energy into the hohlraum wall. Parametric laser-plasma instabilities in the hohlraum plasma can backscatter significant fraction of the laser light by e.g. stimulated Brillouin scattering (SBS) and stimulated Raman scattering (SRS) resulting in reduced efficiency and radiation symmetry. The study and understanding of these processes is thus important to the success of inertial confinement fusion.

In recent years, the understanding of laser-plasma interactions has made significant advances $^{3}$. These measurements require an accurate measurement of laser backscattering from the targets. At the Omega laser two of the 60 beams are equipped with comprehensive backscatter stations comprising each a Full-Aperture Backscatter Station (FABS) and a Near Backscatter Imager (NBI). Light that is backscattered into the final focusing lens aperture is separated from the incoming beam path by a full aperture uncoated glass wedge and down-collimated into the FABS, measuring energy and timeresolved spectra. The NBI measures the energy of the light backscattered outside the focusing lens. This light is diffusely scattered by Spectralon ${ }^{\circledR}$ (Labsphere) coated plates surrounding the beam aperture and an image is recorded with a CCD camera. Both FABS and NBI cover two separate spectral ranges of $351 \pm 10 \mathrm{~nm}$ and $400-700 \mathrm{~nm}$, respectively. 
In this paper we describe a calibration system that was developed to calibrate the energy response of this diagnostic in order to enable accurate absolute measurements of the backscattered energy.

\section{Description of the calibration system}

The approach to the backscatter calibration system is to use a dedicated pulsed laser to measure in-situ the total transmission of the optical path through the FABS, as well as the local point response (sensitivity) of the NBI system. This approach was successfully applied to calibrate the NBI during the NIF early light campaign ${ }^{4}$. A schematic of the pulsed laser calibration system is shown in fig. 1 . The system employs a commercial pulsed Nd:YAG laser (Minilite II, Continuum, Inc.) capable of delivering pulses of 3-5 ns duration and energies of up to $25 \mathrm{~mJ}$ at the $2^{\text {nd }}$ harmonic $(532 \mathrm{~nm})$ and $8 \mathrm{~mJ}$ at the $3^{\text {rd }}$ harmonic $(355 \mathrm{~nm})$. This allows calibration measurements in both spectral ranges of the diagnostics. A fixed fraction $(\sim 10 \%)$ is reflected by a plane-parallel un-coated glass plate

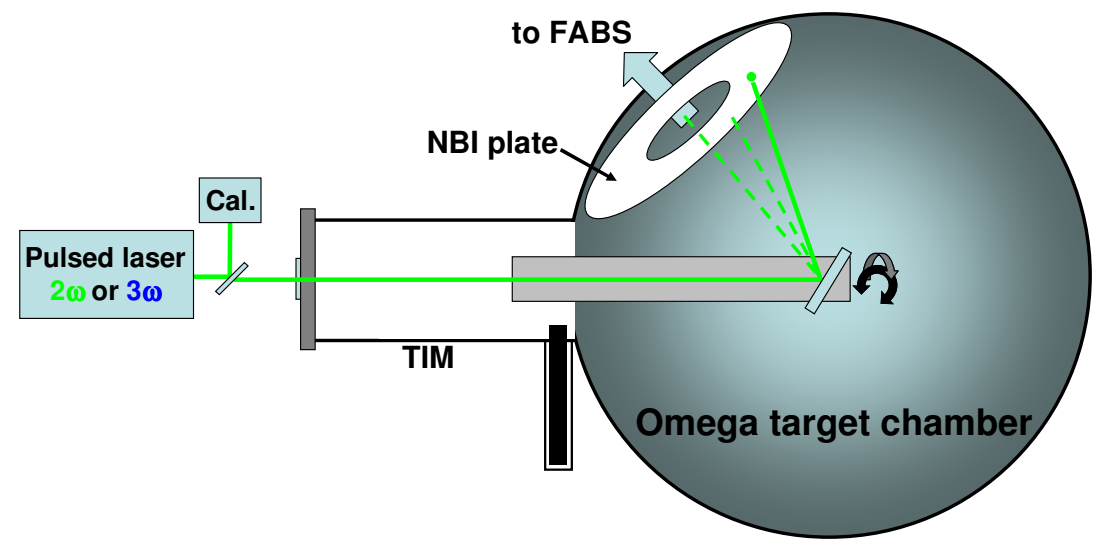

Fig. 1: Schematic of the backscatter calibration system. The pulsed laser is injected through the TIM and the beam is deflected from the target chamber center onto the backscatter diagnostics. 
onto an energy sensor, allowing the energy of each individual pulse to be measured. The beam is injected into the target chamber through a UV-grade Fused Silica window in the back end of one of Omega's ten-inch-manipulators (TIM). The TIM is loaded with an extension carrying a motorized mirror mount which is driven to the center of the target chamber. A 2"-diameter mirror steers the laser beam from the target chamber center onto the NBI scatter plates and into the FABS aperture, respectively.

Having the calibration beam originate from the actual target location is required to propagate the beam down the FABS optical path. Also, this way the NBI response is measured with the beam incident on the scatter plates at the same angle as the backscattered radiation to be measured. While newly refurbished NBI plates have a near ideal Lambertian angular scattering characteristic, older plates that have accumulated a certain amount of target debris, are expected to exhibit a strong component of specularly reflected light, making calibration measurements with the calibration light coming from the actual target location mandatory.

By steering the beam onto a dedicated absolutely calibrated calorimeter the pick-off energy sensor is cross-calibrated.

\section{Calibration measurements}

\section{NBI calibration}

The NBI is calibrated by sending individual pulses onto the NBI plate and acquiring an NBI image with the same configuration as on a real target shot. The motorized mirror is used to scan the calibration laser spot across the plate to measure the spatial variations in the sensitivity. Of order 100 shots are used for a typical calibration run. Fig. 2 shows a 

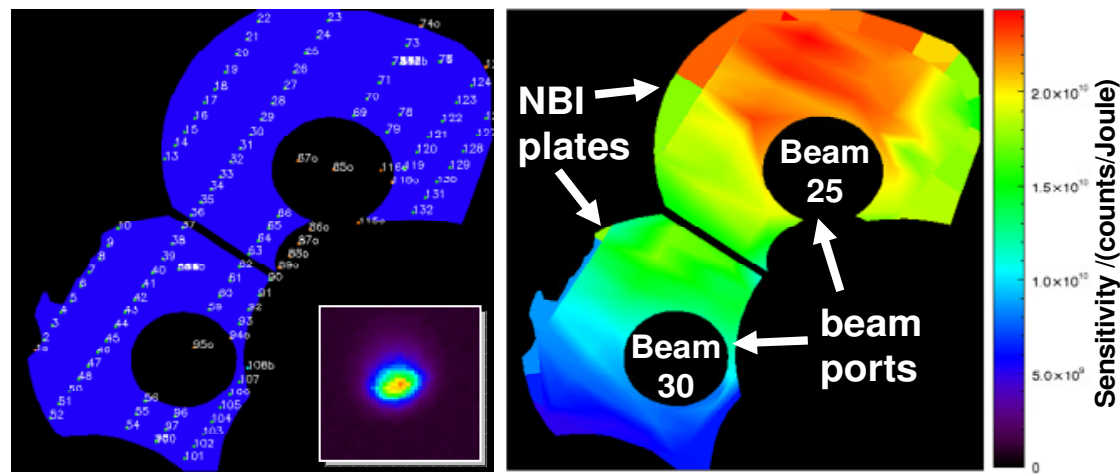

Fig. 2: (left) Shot locations on the NBI plate from a calibration run. The insert show a typical spot from the calibration laser. (right) Sensitivity map for the SRS channel.

typical result from a calibration run. The calibration laser produces a $\sim 15 \mathrm{~mm}$ diameter spot on the NBI plate, corresponding on average to $\sim 10$ pixel in the NBI image. An automated post-processing software locates the spot in each image. Various consistency checks are performed to verify that the spot was correctly recognized and to discard e.g. saturated or clipped laser spots. Then, the local background is subtracted and the total number of counts in the spot is normalized to the laser pulse energy of the respective shot, yielding the local point response (unit counts/Joule). Bi-linear interpolation is used to generate a continuous sensitivity map. The absolute accuracy in the determination of the point response is limited by the absolute calibration of the calorimeter used to crosscalibrate the pick-off energy sensor, and is estimated to $\pm 5 \%$. The relative shot-to-shot reproducibility is approx. $\pm 1 \%$, limited by the shot-to-shot accuracy of the pick-off energy sensor. Error due to camera noise is negligible, as the background RMS is $<2$ counts, compared to the average signal of $\sim 500$ 
counts/pixel over $\sim 100$ pixel.

At the Omega laser, more than 1000 target shots are delivered per year, with targets varying greatly in total mass and materials. This results in a considerable amount of target debris deposited on the NBI plates. As a consequence, the plates are exchanged approx. twice per year. We have performed NBI calibrations on several plates and at various times during the lifetime of the plates. Fig. 3 shows the sensitivity in the shortwavelength channel (at $355 \mathrm{~nm}$ ) over a period of 200 days. The calibrations were performed on various plates and at various instances within their time of usage. We find that the sensitivity decreases significantly as function of time since replacement. A simple model that assumes that debris is deposited at a constant rate of areal density $1 / \tau$ on the plate and that the sensitivity of the plate when completely covered with debris

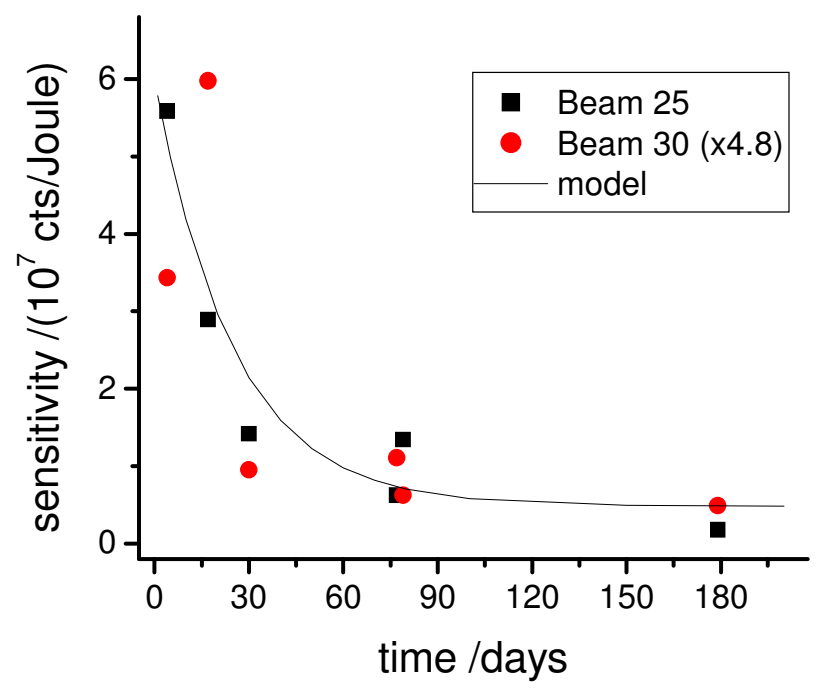

Fig. 3: Variation of NBI sensitivity (at $355 \mathrm{~nm}$ ) with time from last plate change. The sensitivity drops by over $90 \%$ over a few months. The lower sensitivity of Beam 30 is due to the oblique viewing angle of the NBI cameras. 
decreases to a fraction of $\delta$ of the initial sensitivity $S_{0}$, results in the sensitivity decreasing with time as

$$
S=S_{0}\left[(1-\delta) \cdot \exp \left(-\frac{t}{\tau}\right)+\delta\right]
$$

The rate $1 / \tau$ will depend strongly on the frequency of target shots and the amount/mass of target material per shot, while $\delta$ will depend on the target materials used. We find that the calibration data can be reasonably well fitted with $\tau \sim 30 \ldots 40$ days and $\delta \sim 0.02 \ldots 0.1$. For the long wavelength channel $(400-700 \mathrm{~nm})$ we also find a strong decrease in sensitivity over time, the available data is consistent with the same values for the fitting parameters $\tau$ and $\delta$. This shows clearly that only a fairly recent calibration ensures that accurate energy measurements can be performed with the NBI.

Debris on the NBI-plates is a potential source of cross-talk from the short to the long wavelength channel, e.g. due to fluorescence in the 400-700 nm spectral range. By acquiring NBI images on the long wavelength channel while running the calibration laser at $355 \mathrm{~nm}$ we measured a cross-talk of $3 \times 10^{-5}$ on a NBI plate that had been in use for $\sim 80$ days. This amount of cross-talk is completely negligible as it is far below the detection threshold of the diagnostics. However, we note that the fluence of the calibration beam on the NBI plates is only $\sim 5 \mathrm{~mJ} / \mathrm{cm}^{2}$. In a typical experiment, the UV fluence on the NBI plates can reach values of order $100 \mathrm{~mJ} / \mathrm{cm}^{2}$, a level at which fluorescence and therefore cross-talk might be considerably stronger.

\section{FABS calibration and polarization sensitivity}

In the short wavelength channel the FABS is usually calibrated using the Omega laser itself, by firing the opposing $351 \mathrm{~nm}$ beam with an energy of 10-30 J into the FABS 
aperture. However, the final turning mirrors do not transport unconverted $2 \mathrm{w}$ light, which could be used to calibrate the long wavelength channel. We therefore used the pulsedlaser calibration system for an in-situ measurement of the transmission of the FABS optical path at $532 \mathrm{~nm}$. Pulses of $\sim 20 \mathrm{~mJ}$ energy were sent from the target chamber center into the FABS, and the transmitted energy was measured by an absolutely calibrated energy sensor (J9LP, Coherent Inc.). We measured the transmission to $(2.9 \pm 0.3) \times 10^{-3}$. The error is dominated by the absolute calibration of both the energy sensors after the motorized mirror and at the end of the FABS beam path. The measured value lies $\sim 20 \%$ below a simple estimate of the FABS transmission using the properties of the individual optical components. The measured transmission, together with the optical-to-electrical calibration factor of the FABS calorimeters, yields the FABS calibration.

Using a half-wave plate at the exit of the calibration laser we varied the beam polarization to determine the polarization dependence of the FABS transmission. At 532 $\mathrm{nm}$, the transmission was maximized at the polarization of the incoming Omega target beam, with a 2:1 ratio to the perpendicular polarization. This effect is most likely dominated by the reflectivity of coating of the final turning mirror, which is optimized for a wavelength of $351 \mathrm{~nm}$. As expected, using the calibration laser at the $3^{\text {rd }}$ harmonic showed no polarization dependence of the FABS transmission.

\section{Operation}

Fig. 4 shows data from the NBI obtained in an experiment studying the hohlraum energetics employing multiple-ion species plasmas ${ }^{5}$. In these shots the hohlraum gas composition was varied to study the effect of ion Landau damping on laser backscatter. 

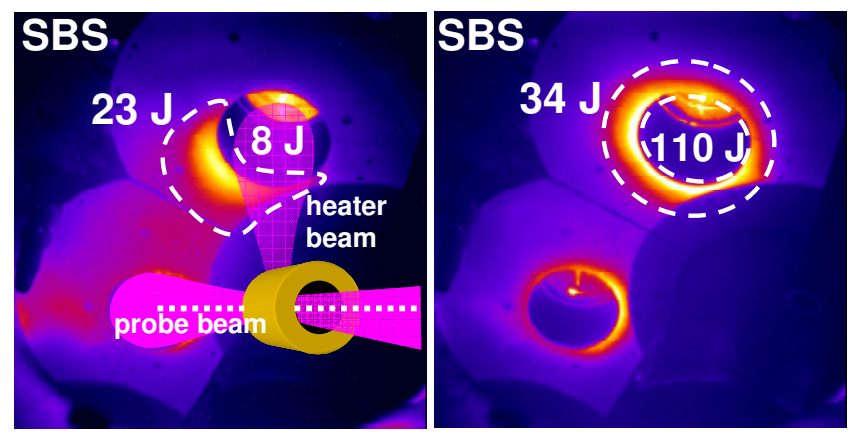

Fig. 4: NBI data in the short wavelength channel, showing strong backscatter from Stimulated Brillouin Scattering. The sketch shows the alignment of the hohlraum using one beam as on-axis probe beam. The energies measured by FABS and on the NBI plates are denoted in the image.

The images demonstrate the range of energies covered by NBI. For the probe beam the energy onto NBI is only about $0.05 \ldots 0.1 \mathrm{x}$ the energy into FABS. The heater beam however encounters higher electron densities and gradients and thus shows significant beam spray and deflection, resulting in energies of $0.3 \mathrm{x}$ up to even $2 \mathrm{x}$ the energy in FABS. An accurate NBI calibration is therefore mandatory to reliably measure backscattered energies.

\section{Acknowledgements}

The authors would like to thank the Omega laser management and operations team for their support in implementing and fielding of the calibration system. This work was performed under the auspices of the U.S. Department of Energy by Lawrence Livermore National Laboratory under Contract DE-AC52-07NA27344.

\footnotetext{
1 John Soures et al., Phys. Plasmas 3, 2108 (1996).
} 
2 John D Lindl et al., Phys. Plasmas 11, 339 (2004).

3 Dustin H Froula et al., Phys. Rev. Lett. 98, 085001 (2007). Dustin H Froula et al., Phys. Rev. Lett. 98, 085001 (2007). Paul Neumayer et al., Phys. Rev. Lett. 100, 105001 (2008). Siegfried H Glenzer et al., Nature Physics 3, 716 (2007). Brian J MacGowan, Phys. Plasmas 3, 2029 (1996).

4 Andrew J Mackinnon et al., Rev. Sci. Instrum. 77, 10E529 (2006).

5 Paul Neumayer et al., Phys. Plasmas 15, 056307 (2008). 